\title{
Epigenetic mechanisms of the karyogenomic system of mature wheat germ derived under conditions of cold stress
}

\author{
E.A. Ivanova*, G.Kh. Vafina \\ Ufa Institute of Biology UFIC RAS, Ufa, Russia
}

DOI 10.18699/ICG-PlantGen2019-27

(c) Autors, 2019

*e-mail: fiona_belobor@mail.ru

\begin{abstract}
In the present work, it was shown that in the process of initiating the growth morphogenesis of mature wheat germ spring (Artyomovka) and the winter derived from it, (Mironovskaya 808), the mechanisms of spatial-temporal reorganization of chromatin structures with the participation of the $A r g-X$ proteolysis function. The features of the proteomic dynamics of bioheteropolymer structures (nucleoplasm, chromatin, nuclear matrix) of interphase nuclei in periods $\mathrm{G}_{1}(\mathrm{Oh} \rightarrow 3 \mathrm{~h} \rightarrow 6 \mathrm{~h}$ ) revealed a change in the localization of the $A r g-X$ zones of proteolytic activity in histones and nonhistones, which can to be associated with changes in the density of tension and changes in the structure of chromatin and to represent the epigenetic mechanisms of the karyogenic system of mature wheat germ, derived under conditions of cold stress.

Key words: Triticum aestivum L.; cold stress; cell nucleus; nucleoplasm; chromatin; nuclear matrix; non-histones; histones; Arg- $X$ (arginine-protein)-processing.
\end{abstract}

\section{Introduction}

According to the priority directions in the study of agricultural plants put forward in 2011 by EPIC, an international consortium of plant epigenetics for the next decade, included a paragraph of the need to understand the molecular basis of the interaction of genotype and environment, change the characteristics of plants in different conditions (Epic Planning Committee, 2012). It is believed that "the chromatin matrix is a physiologically important substrate on which remodeling and the transcription mechanism are deployed" (Allis et al., 2007). The most significant discoveries currently include finding out the location of chromosomes in interphase nuclei and that their structure is controlled by both genetic and environmental factors (Pawlowski, 2010; Tiang, et al., 2012). In order to understand the basic features of these patterns, effective information and computing technologies are being developed. In some of these works, their block-modular arrangement is virtually distinguished, where the blocks of gene networks interconnected by signaling molecules form a hierarchical structure. Currently, methodological progress has greatly advanced the understanding of the molecular-genetic organization of the interphase nucleus. It becomes obvious that the functional dynamics of the domain topology of interphase chromatin is involved in controlling the regulation of various interrelated basic processes in certain regions of the nucleus. We assumed that one of the mechanisms in the reorganization of the chromatin matrix can perform $A r g-X$ protease processing. This assumption is based on the fact that the chromatin of the nucleus is rich in arginine, and, of all the amino acids, only it is able to bind to certain purine and pyrimidine bases of DNA. The purpose of this work was to examine the karyogenomic analysis of the localization of $\operatorname{Arg}-X$ processing in topologically associated super-blocks of the hexaploid system of interphase chromatin in mature wheat germ adapted to cold stress.

\section{Mateials and methods}

The object of the study was the seeds of wheat superelite (Triticum aestivum L.) varieties: Artemovka (spring) and derived from it, Mironovskaya 808 (winter), obtained from the collection of the All-Russian Institute of Plant Industry named. N.I. Vavilova. Experimental work was carried out on the basis of our own patents: 1) on the assessment of the morphophysiological state of the nucleated embryos, 2) cell nuclei isolated from them and 3) supramolecular structures, and 4) non-histone ( $\mathrm{Ngb}$ ) and histone proteins, in which, 5) lacalization of $\mathrm{Arg}-X$ protease processing was revealed. The methodical part of the work is presented in detail in the articles (Ivanova et al., 2014; 2015; Ivanova, 2017). The condition of air-dry seed and embryo (in a state of biological rest), we conditionally took for $0 \mathrm{~h}$. Seed soaking was carried out for 3 hours. The end of this period is marked as $3 \mathrm{~h}$. From air-dried seeds $(0 \mathrm{~h})$, swollen $(3 \mathrm{~h})$ and germinated for $6 \mathrm{~h}$ initiated to grow, separated from the endosperm, cell nuclei were isolated from which supramolecular structures were fractionated into a "labile chromatin" nucleoplasm (Np), chromatin fragile (Chr-I) and firmly (Chr-II) associated with the nuclear matrix (NM) and the NM itself. The $A r g-X$ protease activity in the suprastructures of cell nuclei was assessed by the cleavage of the $\operatorname{Arg}-X$ bonds in the arginine-enriched protein, protamine Salmine-A-I ("Merk").

\section{Results and discussion}

Currently, some progress has been made in the study of chromatin structure on the scale of a whole nucleus. In this paper, the study of the features of the structural reorganization of the interphase chromatin $\mathrm{G}_{1}$ phase of the cell cycle of spring and winter wheat was conducted against the background of the formed physiological features of the seeds of their mature embryos. It is known that the winter variety Mironovskaya 808 was derived by the method of multiple-group selection 
of highly productive, morphologically aligned homogeneous plants from the Artemovka variety (with exact indication of the best families, minimum temperature on the soil surface during wintering over 7 years). The result was that, up to the present, the Mironovskaya 808 is considered a masterpiece of world selection.

There are three regulatory levels in eukaryotic chromatin or genome. The first presents three classes of genetic elements that regulate gene blocks: 1) LCR (locus control regions); 2 ) enhancers, promoters; 3 ) insulators. The second level is chromatin, where regulation is carried out by modifying the crustal histones. At this level, the ATP-dependent chromatin remodeling system and the corresponding enzymes are involved. The first and second levels are interrelated. The third is the level of organization of the interphase core - 3D. It is proved that the nuclear level chromosomes are not randomly located inside the nucleus, each of them occupies a certain territory. It is noteworthy that unstructured amino-terminal tails that carry the guanidine group of arginine-enriched $(\mathrm{H} 3+\mathrm{H} 4)$ and moderately lysine-enriched $(\mathrm{H} 2 \mathrm{~A}+\mathrm{H} 2 \mathrm{~B})$ histones that make up the nucleosome protrude from the nucleosome core. These tails undergo active modification and internucleosomal interactions.

In space-time intervals, heteropolymer blocks were selected on the protein surface of which the interphase chromatin reorganization occurred and the chromatin matrix conformation changed, with the participation of the $\operatorname{Arg}-X$ protease system responsible for protein folding. It is possible that the foregoing is associated with epigenetic mechanisms under the influence of environmental factors.

The $\operatorname{Arg}-X$ protease system of Ngb and histone blocks of supramolecular structures is silent in the suprablocks of the total karyogenomic chromatin matrix of winter wheat mature germ nuclei during $0 \mathrm{~h}$ physiological state.However, in the process of seed swelling (3h), $A r g-X$ of protease-sensitive zones in the linker histone- $\mathrm{HI}$ is exposed at the level of the nuclear matrix and in the Ngb chromatin of unbroken (Chr-I) with NM.

During the period of morphogenetic processes activation in total chromatin suprablocks (6h), Ngb exhibits enhanced exposure at the NM, Chr-I, Chr-II level, as well as in the core histones $\mathrm{H} 2 \mathrm{~A}+\mathrm{H} 2 \mathrm{~B}$ of the nuclear matrix; in contrast to the spring variety Artyomovka, where the relatively uniform exposure of $\mathrm{Arg}-\mathrm{X}$ activity of the $\mathrm{H} 3+\mathrm{H} 4$ histone core occurs only in the Hn nucleoplasm $(0 \mathrm{~h} \rightarrow 3 \mathrm{~h} \rightarrow 6 \mathrm{~h})$. Nucleoplasm performs active nuclear-cytoplasmic relations (due to signaling systems entering the nucleus) and is rich in chaperones that participate in the assembly of nucleosomes. It is possible that the labile chromatin system is the physiological basis of functioning in the spring wheat variety. $A r g-X$ manifestation of nuclear activity at the NM level is explained by the fact that the nuclear matrix is considered as an active dynamic structure that participates in the formation of large enzymatic and regulatory complexes controlling the topology and DNA function.

Not only purely cognitive, but also practical results for biotechnology are expected from progress in understanding morphogenesis. Perhaps the molecular-genetic mechanisms of adaptation of winter wheat are associated with the organization of the nuclear matrix, where $A r g-X$ and hypersensitive sites are formed. Maybe this: LCR - locus control regions. As it is known, an organism is an integrated network of biochemical processes that are in constant dynamics and change as a result of exposure to internal and external conditions. In this regard, supramolecular descriptions of morphogenetic processes are valuable because they already integrate the interactions of many macromolecules and the mechanisms of epigenetic regulation.

\section{Conclusions}

In the present work, it was shown that in the process of initiating the growth morphogenesis of the mature wheat germs of the spring Artyomovka and the winter derived from it, Mironovskaya 808, the mechanisms of spatial-temporal reorganization of chromatin structures with the participation of the $A r g-X$ proteolysis function. Features of the proteomic dynamics of bioheteropolymer structures (nucleoplasm-labile chromatin, fragile and strongly bonded with NM, and also NM itself) interphase nuclei, in periods $\mathrm{G}_{1}(0 \mathrm{~h} \rightarrow 3 \mathrm{~h} \rightarrow 6 \mathrm{~h})$ of the cell cycle phase, revealed a change in the localization of the $\operatorname{Arg}-X$ zones of proteolytic activity in histones and nonhistones, which may be associated with changes in the density of tension and changes in the structure of chromatin and represent the epigenetic mechanisms of the karyogenomic system of mature wheat germ, derived under conditions of cold stress.

\section{References}

Allis C.D., Jenuwein T., Reinberg D. Epigenetics. 2007. Cold Spring Harbor, New York, USA.

Ivanova E.A. $\operatorname{Arg}-X$ proteo-processing as model system for organization of karyogenomics Interphase chromatin of mature germs of wheats, formed in the conditions of cold stress. J. Stress Physiol Biochemistry. 2017;13(4);65-73 (In Russian).

Ivanova E.A., Vafina G.H., Ivanov R.S. Analysis of $A r g-X$ proteasesensitive sites localization in the dynamics of suprastructures of interphase chromatin during induction of mature spring and winter wheat growth morphogenesis. Physiol Plant Genet (Kiev). 2014; 46(3):202-211.

Ivanova E.A., Vafina G.H., Ivanov R.S. Initial morphogenetic features of proteome of suprastructures of interphase chromatin for germination of mature germs in conditions of adapting to winter in wheat. J. Stress Physiol Biochemistry. 2015;11(4):29-42. (In Russian).

Pawlowski W.P. Chromosome organization and dynamics in plants. Plant Biol. 2010;13:640-645. DOI 10.1016/j.pbi.2010.09.015.

The EPIC Planning Committee. Reading the Second Code: Mapping Epigenomes to Understand Plant Growth, Development, and Adaptation to the Environment. The Plant Cell. 2012;24:2257-2261. DOI 10.1105/tpc.112.100636.

Tiang C.L., He Y., Pawlowski W.P. Chromosome organization and dynamics during interphase, mitosis, and meiosis in plants. Plant Physiol. 2012;158:26-34. DOI 10.1104/pp.111.187161.

Acknowledgements. The work is supported and performed as part of the state assignment of the Ministry of Education and Science of Russia No. 075-00326-19-00 on the topic No. R \& D of AAAA-A18-118022190104-7.

Conflict of interest. Authors declare no conflict. 